characteristic flat nasal bridge, mid face hypoplasia, with a disproportionally large head to birth weight and long tapered fingers.

The multidisciplinary team aim to maximize the mobility and functional capabilities of these patients to enable them to achieve normal developmental milestones at their own pace, while improving bone strength through a programme of regular bisphosphonate infusions.

\section{P36 CHRONIC RECURRENT MULTIFOCAL OSTEOMYELITIS, A DIAGNOSIS NOT TO MISS}

Munir Ahmed, Cheuk-Hei Christopher Tong*. Worcestershire Royal Hospital, Worcester, UK

10.1136/archdischild-2019-epa.391

Introduction Chronic Recurrent Multifocal Osteomyelitis (CRMO) is classified as an auto inflammatory disorder. ${ }^{1}$ It mainly affects children and adolescents. There are several diagnostic approaches described in current literature. Roderick et al formulate the Bristol diagnostic criteria, ${ }^{2}$ which is based on combination of clinical and radiological findings. CRMO is characterized by episodic skeletal pain and swelling, associated with systemic symptoms such as fever and malaise. $^{3}$ Plain radiograph demonstrates lytic destruction, with sclerosis within the metaphysis of bone. STIR MRI demonstrates oedema in bone marrow, with lytic lesions and periosteal reaction seen occasionally. ${ }^{4}$ The main stream of management is symptomatic relief using mainly non-steroid anti-inflammatory drugs (NSAID). Most cases of CRMO reported are self-limited and resolve without significant complications. $^{5}$

Case description A 5 year old fit and well boy had a febrile illness lasting for a month. He also complained of anorexia, weight loss and abdominal pain. He also had cervical lymphadenopathy. His symptoms were unresponsive to antibiotics. There was no evidence of infections after investigations. Throughout the next year, he had recurrent fever and started to have lower limb tenderness. He was admitted on multiple occasions. He was reviewed by different sub-specialist and investigated thoroughly. He never had raised inflammatory markers or presence of auto-antibodies. On his whole body MRI scan, he was found to have hyper intensity involving the metaphysis of his upper and lower limb bones. Diagnosis of CRMO was then confirmed by a specialist from tertiary hospital on bone biopsy of lytic lesions.

Discussion CRMO is an uncommon diagnosis, cases are reported to have months to years' time interval between presentation to diagnosis (2). Through this case, we aim to raise awareness amongst clinicians to shorten time to make diagnosis and to avoid unnecessary admissions.

\section{REFERENCE}

1. Hedrich CM, Hofmann R, Pablik J, Morbach H, Girschick HJ. Autoinflammatory bone disorders with special focus on chronic recurrent multifocal osteomyelitis (CRMO). Pediatr Rheumatol Online J. 2013;11:47

2. Roderick MR, Shah R, Rogers V, Finn A, Ramanan AV. Chronic recurrent mltifocal osteomyelitis (CRMO) - advancing the diagnosis. Pediatric Rheumatology (2016) $14: 47$

3. Jurik AG. Chronic regional multifocal osteomyelitis. Semin Musculoskelet Radio 2004; 8:243-253

4. Iyer RS, Thapa MM, Chew FS. Chronic Recurrent Multifocal Osteomyelitis: Review. ARJ 2011; 196:S87-S91

5. Catalano-Pons C, Comte A, Wipff J, et al. Clinical outcome in children with chronic recurrent multifocal osteomyelitis. Rheumatology 2008; 47: 1397-1399 IN THE UK!!

Fatemeh Jenabi*, Alice Leahy, Nicola Foulds, Hans de Graaf. University Hospital Southampton NHS Foundation Trust, Southampton, UK

\subsection{6/archdischild-2019-epa.392}

Background Ehlers-Danlos Syndrome (EDS) is a heterogeneous group of collagen disorders. There is controversy regarding clinical diagnosis of hypermobility EDS. The Beighton score and the recent international classification of EDS are not validated in children. Clinicians get the impression that there is a substantial increase in frequency of referrals with ?EDS especially for musculoskeletal pain.

Methods In this retrospective cohort study we searched local databases of paediatric rheumatology and genetics department for referrals to the paediatric rheumatologist due to concerns regarding having hypermobile EDS. All clinic letters were carefully examined and data collected for patients age, gender, reasons for referral, diagnosis made by rheumatologist and the management plan. We did a descriptive analysis using population proportion confidence interval.

Results In the paediatric rheumatology department we identified 53 referrals (34 female), median age 11 years (range 9 months - 17 years) from June 2017 to June 2018. 59\% had anxious parents with one or multiple psychosocial issues as well experiencing musculoskeletal pain. 12 patients had a family history of hypermobile EDS, three patients were diagnosed with hypermobile EDS in a private clinic, for three patients the GP had suggested EDS, one patient had possible skin features of EDS, parents of two patients had concerns following surfing the internet and one patient had a cardiac and vascular problem possibly related to EDS. The patients were diagnosed being a healthy child (49\%), or having hypermobility $(28 \%)$, arthralgia (8\%), mechanical pain $(7 \%)$, chronic pain (6\%) and classical EDS (2\%). Management included reassurance and discharge (21\%), referral to physiotherapy (76\%), referral to psychology (13\%), referral to other medical teams (21\%). Of all patients $11 \%$ were followed up after the initial review. The genetic department had total of 245 referrals From January 2017 to June 2018. The referral included 62 patients with ?EDS (19 paediatrics, 43 adults), 14 with ? hypermobility (12 paediatrics, two adults) and 169 hypermobile patients with ?Marfan syndrome (44 paediatrics, 125 adults). Most referrals did not have a significant family history or clinical features.

Conclusion Particular attention should be devoted to education and information in first line health care as well as to more effective management of musculoskeletal pain and parental anxiety.

\section{P38 OUR EXPERIENCE IN RESTORING HIP STABILITY IN CHILDREN WITH CEREBRAL PALSY}

${ }^{1}$ Aleh Sakalouski, ${ }^{1}$ Mikhail Herasimenka, ${ }^{2}$ Raman Klimau*, ${ }^{3}$ Leanid Hlazkin, ${ }^{3}$ Mikhail Mikhovich. ${ }^{1}$ Republican Scientific and Practical Centre for Traumatology ans Orthopaedics, Minsk, Belarus; ${ }^{2}$ Brest Regional Children's Hospital, Brest, Belarus; ${ }^{3}$ Mogilev Regional Children's Hospital, Mogilev, Belarus

\subsection{6/archdischild-2019-epa.393}

Introduction and Objective Hip instability in children with cerebral palsy (CP) is a serious progressive problem. 